\title{
A new adenovector system for implementing thymulin gene therapy for inflammatory disorders
}

\author{
María F. Zappa-Villar ${ }^{\mathrm{a}}$, Micaela López-León ${ }^{\mathrm{a}}$, Joaquín Pardo ${ }^{\mathrm{a}}$, Mariana Costa ${ }^{\mathrm{a}}$, Rosana Crespo ${ }^{\mathrm{a}}$, \\ Mireille Dardenne $^{\mathrm{b}}$, Rodolfo G. Goya ${ }^{\mathrm{a}}$, Paula C. Reggiani ${ }^{\mathrm{a}, *}$

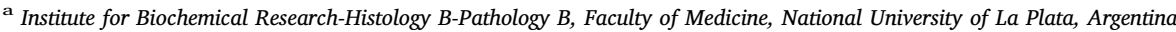 \\ b CNRS UMR8147, Paris Descartes University, Paris, France
}

\section{A R T I C L E I N F O}

\section{Keywords:}

Thymulin

Anti-inflammatory peptide

Gene therapy

Regulatable expression

\begin{abstract}
A B S T R A C T
Thymulin is a thymic peptide possessing anti-inflammatory effects. In order to manipulate thymulin expression in gene therapy studies, we built a bidirectional regulatable two-vector Tet-Off system and the corresponding control system. The experimental two-vector system, ETV, consists of a recombinant adenovector (RAd) harboring an expression cassette centered on a Tet-Off bidirectional promoter flanked by a synthetic gene for thymulin and the gene for humanized Green Fluorescent Protein (hGFP). The second adenovector of this system, RAd-tTA, constitutively expresses the regulatory protein tTA. When cells are co-transduced by the two adenovector components, tTA activates the bidirectional promoter and both transgenes are expressed. In the presence of the antibiotic doxycycline (DOX) transgene expression is deactivated. The control two-vector system, termed CTV, is similar to ETV but only expresses hGFP. In CHO-K1, BHK, and C2C12 cells, ETV and CTV induced a dose-dependent hGFP expression. In CHO-K1 cells, transgene expression was almost completely inhibited by DOX (1 mg/ml). After intracerebroventricular injection of ETV in rats, thymulin levels increased significantly in the cerebrospinal fluid and there was high hGFP expression in the ependymal cell layer. When injected intramuscularly the ETV system induced a progressive increase in serum thymulin levels, which were inhibited when DOX was added to the drinking water. We conclude that our regulatable two-adenovector system is an effective molecular tool for implementing short and long-term anti-inflammatory thymulin gene therapy in animal models of acute or chronic inflammation.
\end{abstract}

\section{Introduction}

Thymulin is a thymic hormone that participates in several aspects of intra- and extrathymic T-cell differentiation (Bach 1983). Thymulin is produced by the thymic epithelial cells (Dardenne et al., 1974) and consists of an inactive peptide component termed FTS (a French acronym for serum thymus factor), coupled in an equimolecular ratio to the ion zinc (Gastinel et al., 1984), which confers biological activity to the peptide (Dardenne et al., 1982). The metallopeptide active form is characterized by a specific molecular conformation that has been determined by nuclear magnetic resonance (Cung et al., 1988). There is a growing body of evidence that, in addition to its immunomodulatory actions, thymulin possesses hypophysiotropic activity (Goya et al., 2004). An adenovector expressing a synthetic gene for the thymulin analog methionine-FTS (metFTS), constructed by us, was shown to induce long-term expression of transgenic thymulin in serum when intramuscularly (i.m.) injected in rodents (Reggiani et al., 2006). Moreover, using this vector we demonstrated that neonatal thymulin gene therapy is able to prevent the typical ovarian dysgenesis that occurs in congenitally athymic nude female mice after puberty (Reggiani et al., 2012) and restores their deficient gonadotropin serum levels (Goya et al., 2007). More recent reports revealed an antiinflammatory activity of thymulin in the brain and other organs, which makes thymulin a molecule of clinical interest. Among the immune factors that interact with thymulin are a set of cytokines that play a major role in the inflammatory response. In a model of endotoxininduced neuroinflammation, prior intracerebroventricular (i.c.v.) injections with thymulin $(0.1-1 \mu \mathrm{g})$ prevented the endotoxin-induced hyperalgesia and exerted differential effects on the up-regulated levels of cytokines in different regions of the central nervous system (CNS), suggesting a neuroprotective role of thymulin in the brain (SafiehGarabedian et al., 2003). A thymulin analog also induced a significant

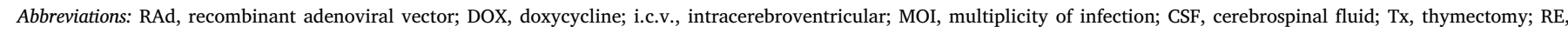
restriction endonuclease; MCS, multiple cloning site; $\mathrm{LV}$, lateral ventricle

* Corresponding author at: INIBIOLP, Faculty of Medicine, UNLP, CC 455, zip 1900, La Plata, Argentina.

E-mail addresses: goya@isis.unlp.edu.ar (R.G. Goya), paulareggiani@conicet.gov.ar (P.C. Reggiani). 
amelioration of endotoxin-induced hyperalgesia and a decrease in the release of the proinflammatory cytokines IL-1ß, IL- 6 , and TNF $\alpha$ in the hippocampus and brainstem. Injected alone, the analog induced an upregulation of IL-10 in the hippocampus and brainstem and produced a marked enhancement of the level of this cytokine in the same areas when endotoxin was simultaneously applied (Safieh-Garabedian et al., 2011). A beneficial action of thymulin was also reported in a number of models of organ-specific inflammation: In alloxan- and streptozotocininduced diabetes, pretreatment with thymulin significantly suppressed hyperglycemia and prevented the destruction of pancreatic beta cells (Yamanouchi et al., 1994). A similar protection was reported in myocarditis induced by encephalomyocarditis virus and in pulmonary hypertension induced by monocrotaline (Henriques-Coelho et al., 2008). In the latter model, thymulin effect was related to the inhibition of the expression of the proinflammatory cytokine IL- 6 and to the suppression of the p38 MAPK pathway. In a more recent report, Da Silva et al. (2014) implemented thymulin gene therapy in a mouse model of allergic asthma. In this study, intratracheal injection of a nanoparticle-complexed plasmid expressing our synthetic gene for thymulin decreased lung eosinophil infiltration, IL-13, eotaxin, transforming growth factor- $\beta$, vascular endothelial growth factor, and increased lung interferon- $\gamma$. The changes were paralleled by a reduction in the fraction area of alveolar collapse, bronchoconstriction index, smooth-muscle-specific actin expression, collagen fiber content in the airway and alveolar septa and airway hyperresponsiveness. This study revealed that DNA-nanoparticle-mediated thymulin gene therapy can modulate the inflammatory and remodeling processes in experimental allergic asthma, therefore improving lung mechanics. The thymulin plasmid used in the above study (Da Silva et al., 2014) revealed the suitability of thymulin vectors for the treatment of inflammatory pathologies. We reasoned that a viral vector expressing thymulin and a reporter gene both under the control of a bidirectional regulatable promoter would allow experimenters, and eventually clinical gene therapists, to manipulate the expression of these genes according to treatment needs. Capitalizing on our expertise in the molecular biology of thymulin we decided to construct such a vector. In the present study, we report its construction and both an in vitro and in vivo characterization of a bidirectional regulatable two-adenovector Tet-Off system expressing humanized GFP (hGFP) and our synthetic gene for thymulin (metFTS).

\section{Materials and methods}

\subsection{Construction of a regulatable bidirectional two-adenovector system}

\subsubsection{Construction of shuttle plasmid pDC516-(GFP-TRE-FTS)}

A variant of our synthetic gene metFTS was constructed (Reggiani et al., 2006), which contained the restriction endonuclease (RE) sites MluI and EcorRV in its $5^{\prime}$ and $3^{\prime}$ regions, respectively. This insert was then cloned into the Multiple Cloning site I (MCS I) of the pBI Tet-Off vector (Clontech (Takara), Mountain View, CA), a bidirectional expression vector harboring the regulatable TRE element flanked by two minimal Cytomegalovirus Promoters (PminCMV) positioned in opposite orientations. Afterwards, the hGFP cDNA was inserted into the MCS II of the above mentioned plasmid in the NotI restriction site. This step was checked for correct orientation by means of restriction mapping. The resulting plasmid was named pBI-(GFP-TRE-FTS). Therefrom, the cassette GFP-TRE-FTS was excised with the REs SalI and BglII and cloned into the corresponding restriction sites of the plasmid pDC516w/p, a modified version of pDC516 (Microbix Biosystems Inc., Mississauga, Canada) by excising its promoter but keeping the polyadenylation signal SV40 pA. In order to provide the second polyadenylation signal to this construct, a DNA fragment coding for the SV40 pA, flanked by the restriction sites for XhoI and XbaI, was inserted into the corresponding restriction sites of our plasmid. The final construct was named pDC516-(GFP-TRE-FTS), which harbors the regulatable bidirectional cassette and the SV40 pA signal downstream both transgenes. The control plasmid, named pDC516-(GFP-TRE-MCS), was constructed following the same procedure, except that the MCS I was left empty, which resulted in a final plasmid only expressing hGFP.

\subsubsection{Construction of shuttle plasmid pDC515-tTA}

In order to construct this plasmid, the DNA sequence coding for the regulatory protein tTA was excised from plasmid pTet-Off (Clontech) into the MCS of the shuttle plasmid pDC515 (Microbix Biosystems Inc.) by using the REs EcoRI and BamHI.

\subsubsection{Generation of recombinant adenoviral vectors ( $R A d)$}

RAds were constructed by a variant of the two plasmid method (Hitt et al., 1998) employing the AdMax plasmid kit (Microbix, Canada) as described elsewhere (Reggiani et al., 2006). Briefly, the kit uses a shuttle plasmid (pDC515 or pDC516) harboring a MCS and FRT recognition sequences for the yeast FLP recombinase. The cassette is flanked by the sequences of the adenovirus type 5 (Ad5) E1 region. The second plasmid of the kit, the genomic plasmid pBHGfrt(del)E1,3FLP, consists of the entire genome of Ad5, containing deletions in the regions E1 and E3. Upstream the E1 deletion, this plasmid contains an expression cassette harboring the gene for yeast FLP recombinase and immediately downstream the E1 deletion, a FRT recognition site. In order to generate the two RAds of our two-adenovector system, the genomic plasmid was cotransfected with either shuttle pDC516-(GFPTRE-FTS) or shuttle pDC515-tTA in Human Embryonic Kidney 293 (HEK293) cells. In cotransfected HEK293 cells, the FLP recombinase is expressed and catalyzes a site-directed recombination of the expression cassette of pDC516-(GFP-TRE-FTS), pDC516-(GFP-TRE-MCS) or pDC515-tTA into the left end of pBHGfrt(del)E1,3FLP, thus generating the genome of the desired recombinant adenoviral vectors. The newly generated RAds, termed RAd-(GFP-TRE-FTS), RAd-(GFP-TRE-MCS) and RAd-tTA were rescued from HEK293 cell lysates and plaque purified. They were further purified by ultracentrifugation in $\mathrm{CsCl}$ gradient. Final virus stocks were titrated by a serial dilution plaque assay.

\subsection{In vitro characterization of the system}

\subsubsection{Optimization of the $\mathrm{MOI}$}

In order to evaluate the performance of the regulatable bidirectional two-adenovector system in vitro, we assessed the expression of the reporter gene hGFP at several MOIs in the following cell lines: mouse muscle myoblasts $\mathrm{C} 2 \mathrm{C} 12$, rat glial B92 cells and Chinese hamster ovary (CHO-KI) cells. These cell lines were used and maintained as recommended by the American Type Culture Collection (ATCC). The MOIs (pfu/cell) 0, 12, 36, 100, 500 and 1000, were tested in triplicate for the experimental two-vector system (ETV: RAd-(GFP-TRE-FTS) + RAdtTA) and the control two-vector system (CTV: RAd-(GFP-TRE-MCS) + RAd-tTA). In every case, equal ratios of the RAd-tTA and RAd ETV/ CTV vectors were used. On experimental day $-1,1.6 \times 10^{5}$ cells were seeded on 24-well plates. On day 0 virus transduction for each cell line was performed in Fetal Bovine Serum (FBS)-free medium for $1.5 \mathrm{~h}$, rocking the plate every $15 \mathrm{~min}$. Afterwards, the medium was replaced by $500 \mu \mathrm{l}$ maintenance medium, which was kept until the end of the experiment. On day 2, microphotographs of the cultures were taken and cells were lysed in order to quantitate GFP expression as described in Section 2.4.2.

\subsubsection{Characterization of system regulatability}

In order to assess in vitro the regulatability of the system by doxycycline (DOX), CHO-KI cells were used. On experimental day -1 cells were seeded on 12-well plates and, when appropriate, DOX (Clontech) was added to the culture media at a concentration of 1 $\mathrm{ug} / \mathrm{ml}$. On day 0 , culture medium was replaced by, a) culture medium as a negative control for virus transduction, b) RAd-tTA and RAd-(GFP- 
TRE-FTS) in culture medium as a positive control for baseline hGFP expression, c) RAd-tTA and RAd-(GFP-TRE-FTS) in medium containing DOX to assess regulatability of the system and, d) RAd-(GFP-TRE-FTS) alone in culture medium as negative control for the two-vector system functionality. In every case we used a MOI $=200$ for each adenovector. On day 2, micrographs were taken in order to observe hGFP fluorescence, supernatant was collected to determine thymulin and cells were lysed to quantify GFP fluorescence.

\subsection{Animals and in vivo procedures}

Young (4-9 week) C57BL/6 male and female mice as well as threemonth-old Sprague Dawley male rats were raised in our animal facilities (INIBIOLP). Animals had free access to food and water and were kept at $22{ }^{\circ} \mathrm{C}$ with a light/dark cycle of $12 / 12 \mathrm{~h}$. All experiments with animals were done following the Animal Welfare Guidelines of NIH (INIBIOLP's Animal Welfare Assurance No A5647-01) and in conformity with the Guidelines on Handling and Training of Laboratory Animals published by the Universities Federation for Animal Welfare (The Biological Council Animal Research and Welfare Panel (Eds.), UFAW, Pert, 1992). The corresponding protocol was approved by our Institutional Animal Welfare Committee, IACUC protocol \# P05-02-2015, 9th October, 2015.

\subsubsection{Transgene expression in skeletal muscle}

On experimental day -14 rats were thymectomized and divided into two groups ( $\mathrm{N}=4$ /group): CTV and ETV. On experimental day 0 , following the procedure described in Section 2.3.5.2, they received $0.1 \mathrm{ml}$ of a suspension containing $1 \times 10^{8}$ plaque forming units (pfu) of each virus component of CTV or ETV, according to the experimental group. In order to assess serum thymulin levels, blood was withdrawn every 7 days as described in Section 2.3.5.4. On day 2, one experimental subject from each group was euthanized to observe hGFP fluorescence (see Section 2.3.5.5). The remainder of the animals was euthanized on post vector injection day 49.

\subsubsection{Thymulin expression regulatability in skeletal muscle}

The animals were divided into two groups ( $\mathrm{N}=5$ /group): CTV and ETV. On day 0 they were injected as described in Section 2.3.5.2. Afterwards, blood was withdrawn every 5 days from their tail veins until day 25 in order to assess serum thymulin levels. On day 15 DOX was added to the drinking water at a final concentration of $2 \mathrm{mg} / \mathrm{ml}$. This preparation was renewed every 2 days until the end of the experiment. On experimental day 20 the drinking water of the rats was supplemented with $\beta$-methasone at a final concentration of $15 \mathrm{mg} /$ 1. The aim of this corticoid addition was to pharmacologically inhibit endogenous thymulin secretion from the thymus (Goya et al., 2003).

\subsubsection{Transgene expression in the brain}

Animals were divided into two groups ( $\mathrm{N}=4$ /group): CTV and ETV. Following the procedure described in Section 2.3.5.3, they were injected in each LV $10 \mu \mathrm{l}$ of a suspension containing $1 \times 10^{9}$ pfu of each virus component of the CTV and ETV systems. Seventy-two h later, CSF was obtained as described in Section 2.3.5.4 to assess thymulin levels. Immediately afterwards, animals were transcardially perfused and their brains processed for fluorescence microscopy as described in Section 2.3.5.6. CSF samples were stored at $-80^{\circ} \mathrm{C}$.

\subsubsection{Time course of hGFP expression in the cerebral ventricles}

The duration of hGFP expression in the ependymal layer of thymectomized animals i.c.v. injected with ETV $(\mathrm{N}=15)$ was monitored. After performing i.c.v. injection, three rats were euthanized on experimental days $2,7,14,21$ and 28 . Their brains were processed for fluorescence microscopy and micrographs taken as described in Section 2.3.5.6.

\subsubsection{Surgical and histological procedures}

2.3.5.1. Thymectomy ( $T x)$. Tx in rats was performed as previously described (Waynforth and Flecknell, 1992). Tx in mice was performed by aspiration with the animals under Avertin (2,2,2 tribromoethanol) anesthesia $(0.4 \mathrm{mg} / \mathrm{g}$ BW, i.p.). In order to ensure the absence of circulating thymulin, Tx animals were studied 14 days after surgery.

2.3.5.2. Skeletal muscle injections. Animals were anesthetized with $2-3 \%$ isoflurane at flow rate of $1.51 / \mathrm{min}$ oxygen and positioned ventrally on the operating table with both hindlimbs extended and gently immobilized. The hair was shaved from the skin covering the tibialis anterior (TA) muscles of both legs; the skin disinfected with topic povidone-iodine and finally a $1-\mathrm{cm}$ long incision was made to access the muscular compartment. The TA muscle of their hindlimbs was identified and $100 \mu \mathrm{l}$ vector was injected in its middle portion.

2.3.5.3. Stereotaxic injections. I.c.v. injections were performed as previously described (Pardo et al., 2016). Briefly, rats were anesthetized with ketamine hydrochloride $(40 \mathrm{mg} / \mathrm{kg}$; i.p.) plus xylazine $(8 \mathrm{mg} / \mathrm{kg} ;$ i.m. $)$ and placed on a stereotaxic apparatus. Injections in the lateral ventricles $(10 \mu$ vector suspension, per side) were performed by placing the tip of a Hamilton syringe needle at the following coordinates relative to the bregma: $-0.8 \mathrm{~mm}$ anteroposterior, $4.1 \mathrm{~mm}$ dorsoventral and $\pm 1.5 \mathrm{~mm}$ mediolateral (Paxinos and Watson, 1998).

2.3.5.4. Blood and CSF collection. At different times, animals were isoflurane anesthetized and their tails immersed in $42{ }^{\circ} \mathrm{C}$ water for $40-50 \mathrm{~s}$ to dilate blood vessels. The tail vein to be bled was located and punctured with a $22 \mathrm{G}$ needle and $2 \mathrm{ml}$ blood was collected using a Kahn tube. Before sacrifice, CSF was obtained from the great cerebral cistern by puncture as previously documented (Nishida et al., 2011). Briefly, rats were anesthetized with ketamine-xylazine and CSF withdrawn with a syringe fitted to a $30 \mathrm{G}$ needle. With this procedure it was possible to obtain $50-100 \mu \mathrm{l} \mathrm{CSF}$.

2.3.5.5. Muscle processing. Two days after transduction with our twovector system, rats were euthanized and the TA muscles were promptly dissected, fixed with phosphate buffered paraformaldehyde 4\% (4\% PBPFA) for $48 \mathrm{~h}$ at $4{ }^{\circ} \mathrm{C}$ and cryopreserved (30\% sucrose in 4\% PB-PFA) for 3 days at $-20{ }^{\circ} \mathrm{C}$. Finally, muscles were embedded in tissue-freezing medium (Cryoplast ${ }^{\circ}$, Biopack, Buenos Aires, Argentina) and stored at $-80{ }^{\circ} \mathrm{C}$ until further processing. Longitudinal $30-\mu \mathrm{m}$ thick sections were obtained with a freezing microtome (Leitz 1320, Wetzlar, Germany) and mounted with fluorescent mounting medium (Fluoromount $\mathrm{G}^{\mathrm{rm}}$, Electron Microscopy Sciences, PA, USA).

2.3.5.6. Brain processing. Animals were placed under deep anesthesia and perfused with 4\% PB-PFA ( $\mathrm{pH} 7.4)$ fixative. The brains were removed and post-fixed in $4 \%$ PB-PFA overnight at $4{ }^{\circ} \mathrm{C}$. Brains were processed for fluorescence microscopy as described elsewhere (Pardo et al., 2016). To obtain fluorescence images, coverslips were covered with Fluoromount $\mathrm{G}$ and mounted onto the slides.

\section{4. hGFP expression}

\subsection{1. hGFP fluorescence image acquisition}

Cell culture images were acquired with an Olympus Digital Camera E-330 attached to Olympus IX-71 inverted fluorescence microscope, whereas hGFP expression in muscle and brain tissue was assessed with an Olympus BX51 microscope equipped with a DP70 CCD Video Camera. The subsequent image processing was performed with (Image-Pro ${ }^{\oplus}$ Plus) software (Version 5.1.2, Media Cybernetics, Inc).

\subsection{2. hGFP quantification in cell lysates}

Cell lysates were collected from 24-well plates as follows: after 
culture medium was removed, the monolayer was rinsed three times with phosphate buffered saline (PBS) and then incubated with $150 \mu \mathrm{l} /$ well lysis buffer (0.1\%, Tritón X-100, $1 \mathrm{mM}$ EDTA in PBS), for $30 \mathrm{~min}$ at $4{ }^{\circ} \mathrm{C}$. Afterwards, the lysates were collected and centrifuged for $1 \mathrm{~min}$ at $13,000 \mathrm{rpm}$. Then, $100 \mu \mathrm{l} /$ well clean supernatant was added in a $\mathrm{Nunc}^{\mathrm{TM}}$ MicroWell $^{\mathrm{TM}}$ 96-Well Optical-Bottom Plates with Polymer Base (Catalog \#:265301, Thermo Scientific $\left.{ }^{\mathrm{TM}}\right)$. Fluorescence intensity was subsequently measured in a multimode microplate reader (Beckman Coulter DTX 880 Multimode Detector) at excitation and emission filter (nm) of 485 SL 1 and 535 SL 1, respectively. The results were expressed as arbitrary fluorescence units.

\subsection{Thymulin bioassay}

Biologically active thymulin was measured in cell cultures and serum by a rosette bioassay described in detail elsewhere (Dardenne and Bach, 1975). This method is based on the ability of thymulin to restore the inhibitory effect of azathioprine $(\mathrm{Az})$ on rosette formation in spleen cells from Tx mice. Briefly, sera or supernatants were filtered through membranes (30 kDa cut-off; YMT micropartition system, PMS-I Amicon Corporation, Denver) and the ultrafiltrates were diluted serially and incubated for $90 \mathrm{~min}$ at $37^{\circ} \mathrm{C}$ with spleen cells from C57BL/6 Tx mice in the presence of $10 \mu \mathrm{g} \mathrm{Az} / \mathrm{ml}$, which is a concentration that inhibits 50\% rosette formation in normal but not thymectomized mice. Rosettes were then allowed to form by centrifugation with sheep red blood cells and counted in a hemacytometer after gentle resuspension. Rosette formation was inhibited by thymulin in a dose-dependent manner. The inhibitory activity of samples was compared with that of a standard curve using synthetic thymulin (a kind gift from P. Heinklein, Humboldt University, Berlin). Values were expressed as bioactive thymulin concentration in the sample. The sensitivity of the assay is $1 \mathrm{fg}$ thymulin/ml.

\subsection{Statistical analysis}

All data are expressed as mean \pm SEM (standard error of the mean). Comparisons between experimental groups were done by unpaired Student's $t$-test or ANOVA followed by Dunnett, Tukey's or Sidak tests.

\section{Results}

\subsection{Structure and function of the two-adenovector system for hGFP and thymulin}

We constructed a two-adenovector system based on the transactivation of the bidirectional expression cassette GFP-TRE-FTS by the regulatory protein tTA that binds to the TRE element of the bidirectional promoter PminCMV-TRE-PminCMV, almost inactive per se, and fully activates the expression of the FTS and hGFP transgenes (Fig. 1A). When DOX is added to the system the antibiotic binds to an allosteric site of the tTA protein inducing a conformational change that causes a loss of affinity of tTA for the TRE element. Thus, the tTA protein dissociates from the promoter, which becomes inactive (Fig. 1B). By themselves RAd-(GFP-TRE-FTS), RAd-(GFP-TRE-MCS), and RAd-tTA are inactive concerning transgene expression.

\subsection{Characterization of the system in vitro}

\subsubsection{Optimization of the multiplicity of infection (MOI)}

The MOI-dependent hGFP expression of the ETV and CTV systems was assessed in three cell lines: CHO-KI, B92 and C2C12. CHO-K1 cells were the most receptive to virus transduction followed by B92 cells. C2C12 cells were less amenable to transduction. This is in line with previous reports stating that $\mathrm{C} 2 \mathrm{C} 12$ myoblasts express low levels of the primary Ad membrane receptor (Kimura et al., 2001). In CHO-KI and
B92 cells, transduction levels were low up to MOI 36 but increased significantly from MOI 100 (Fig. 2). In contrast, C2C12 cells showed a substantial increase starting from MOI 500.

\subsubsection{Thymulin and hGFP regulatability in cultures}

The DOX-dependent regulatability of the ETV system was studied. In CHO-KI cell cultures DOX $(1 \mathrm{mg} / \mathrm{ml})$ induced a complete inactivation of the bidirectional Tet-Off promoter as shown by the almost complete disappearance of thymulin from the cell supernatants and hGFP fluorescence from the cell lysates (Fig. 3, upper and lower panels, respectively). As expected, RAd-(GFP-TRE-FTS) alone was inactive.

\subsection{Characterization of the two-vector system in vivo}

\subsubsection{Thymulin and hGFP expression in the rat brain}

Two days post i.c.v. injection the ETV, but not the CTV, system markedly increased thymulin levels in the cerebrospinal fluid (CSF) (Fig. 4A). Two days after i.c.v. injection, the ETV system selectively transduced the ependymal cell layer, expressing high levels of hGFP fluorescence. The expression of hGFP in the ependymal cell layer of animals i.c.v. injected with the ETV system reached maximal intensity in the LV (lateral ventricle) and third ventricle ( $3 \mathrm{~V})$ from post injection day 2 until day 7, progressively fading afterwards (Fig. 4B). On post injection day 14 hGFP fluorescence in the ependymal cell layer of the $3 \mathrm{~V}$, but not the LV, was still detectable. On post injection day 21 fluorescence was no longer detectable in the cerebral ventricles (Fig. 4B).

\subsubsection{Serum thymulin regulatability}

As expected Tx induced a sharp fall in circulating thymulin levels in the animals. Intramuscular injection of the ETV system in young Tx rats induced a cumulative increase in serum thymulin levels which by post injection day 10 , reached $300 \%$ higher levels than in pre Tx animals, remaining at those levels until the end of the experiment, on post injection day 49 (last day monitored). Serum thymulin levels in Tx rats injected with the CTV system remained at nearly undetectable levels throughout the experiment (Fig. 5A). When the ETV system was i.m. injected in non-Tx young rats, serum thymulin increased, reaching maximal values around post injection day 10 . When DOX was added to the drinking water on post injection day 15, serum thymulin levels rapidly fell to pre injection levels. When $\beta$-methasone was added to the drinking water of the same animals on post injection day 20, basal thymulin levels fell rapidly, reaching near non-detectable levels on day 25. Rats injected with the CTV system showed no changes in thymulin levels until the day $\beta$-methasone was added to their drinking water, falling to near non-detectable levels on day 25 (B).

\section{Discussion}

Although the prospect of implementing thymic hormone gene therapy appears as an interesting avenue of pre-clinical research, none of the genes coding for the known thymic peptides has been cloned. In 2002 it was suggested that a possible way to overcome this problem could be to construct "artificial genes" coding for those thymic peptides whose amino acid sequences are short and require no posttranslational processing (Goya et al., 2002). This was achieved for thymulin, whose DNA sequence was initially cloned in a bacterial plasmid (Calenda et al., 1988). Subsequently, a DNA sequence coding for the biologically active thymulin analog metFTS was constructed and cloned in an adenoviral vector termed RAd-metFTS (Reggiani et al., 2006). When i.m. administered to Tx mice and rats (which possess non-detectable levels of circulating thymulin), RAd-metFTS induced sustained supraphysiological serum levels of biologically active thymulin which remained high for at least 112 days in mice and for up to 270 days in rats. Neonatal thymulin gene therapy showed significant therapeutic efficacy in the reproductive system of congenitally athymic female mice 


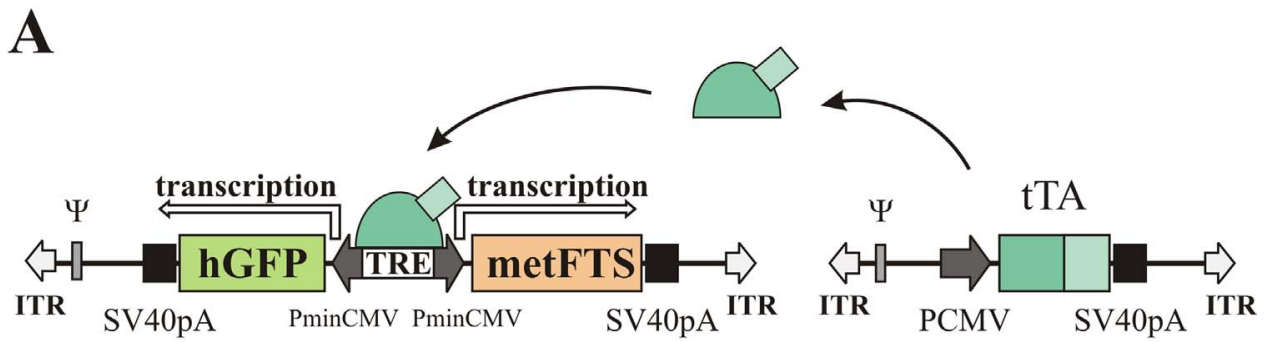

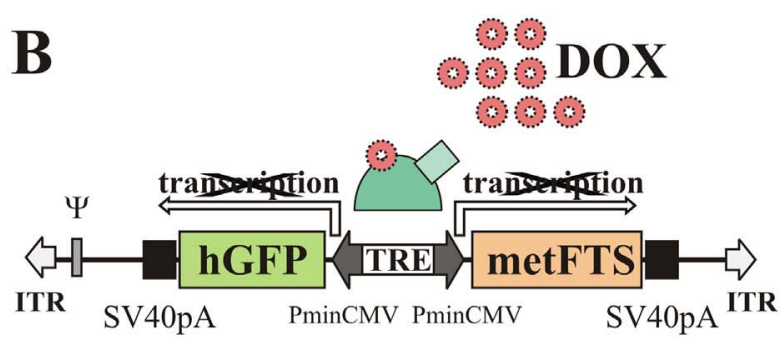

RAd-(GFP-TRE-FTS)

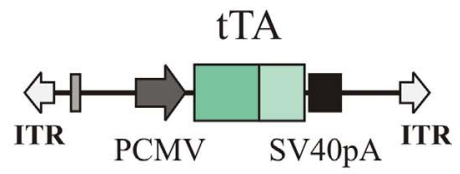

RAd-(tTA)

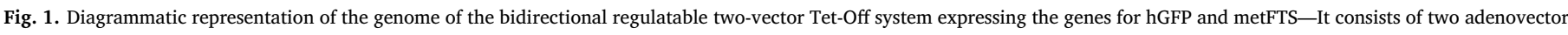

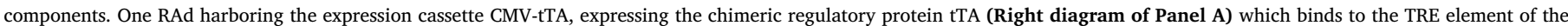

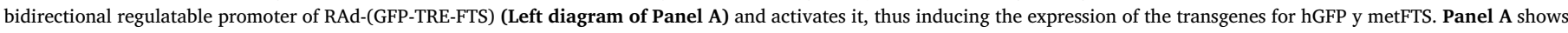

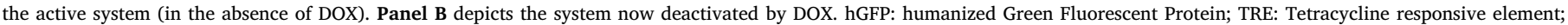

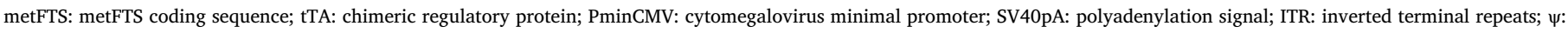
packaging signal.
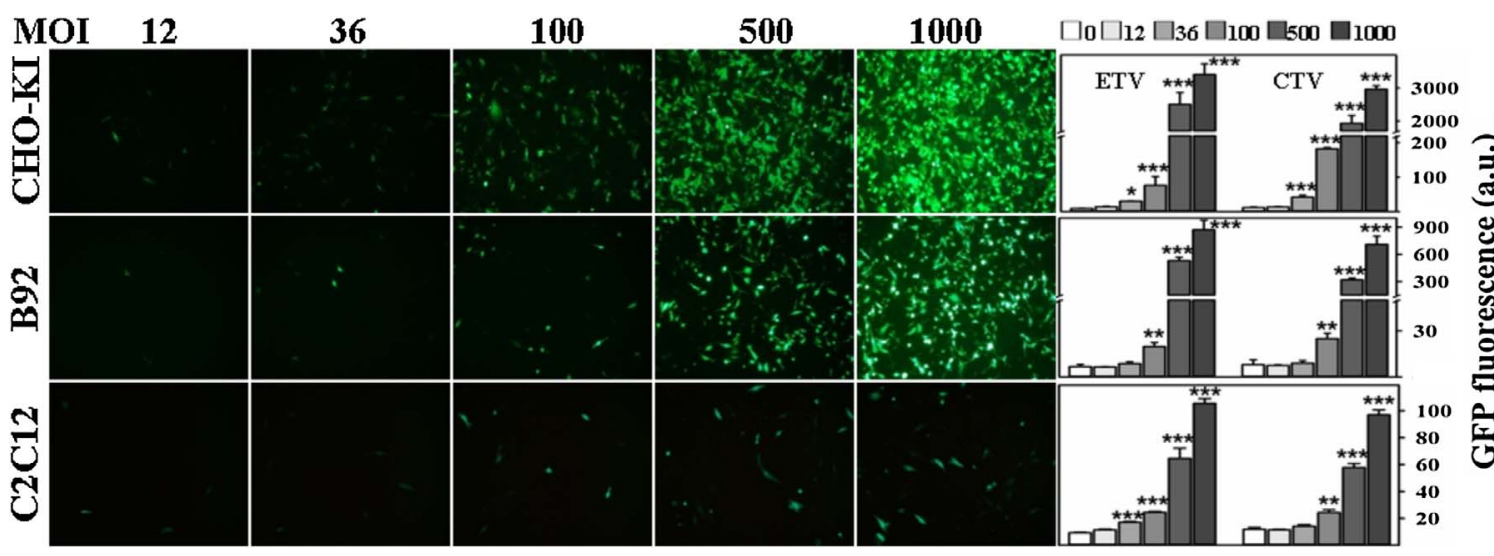

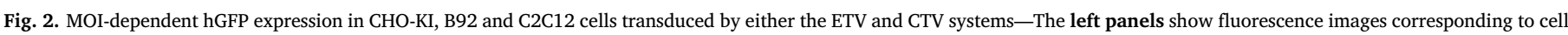

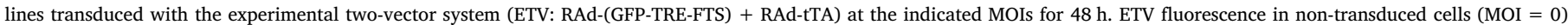

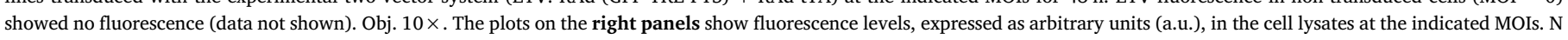

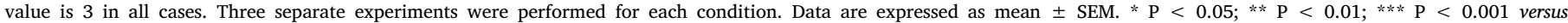
corresponding MOI $=0$ control, analyzed by one-way ANOVA with post-hoc Dunnett test.

\section{(Reggiani et al., 2006).}

In the brain, chronic inflammation is thought to be a potential cause of nigral dopaminergic neurodegeneration in Parkinson's disease (McGeer and McGeer 2004). In this context, it is of interest to seek anti-inflammatory compounds active in the brain and suitable for longterm treatments aimed at protecting nigral neurons from the deleterious effects of pro-inflammatory insults. One such treatment could involve the use of thymulin which is emerging as a promising anti-inflammatory molecule. In effect, thymulin is an immunomodulatory peptide (Safieh-Garabedian et al., 1992) which has been used in the past for the treatment of certain autoimmune pathologies (Bach et al., 1984). More recent studies suggest that thymulin possesses anti-inflammatory activity in the central nervous system (Safieh-Garabedian et al., 2003, 2011).

In the present study, i.c.v. injection of the ETV system selectively transduced the ependymal cell layer and markedly increased thymulin levels in the CSF. Interestingly, adenoviral-mediated expression of metFTS in the substantia nigra and hypothalamus of adult Tx rats had a significantly longer duration (at least 90 days) than adenoviralmediated expression of the gene for GFP or $E$. coli $\beta$-galactosidase in the same brain regions (Morel et al., 2006). This observation is consistent with the anti-inflammatory activity in the brain reported for thymulin and a thymulin analog. Nevertheless, hGFP expression duration of the ETV system was substantially shorter in the ependymal cell layer than the thymulin expression duration of the RAd-metFTS administered in the brain parenchyma (Morel et al., 2006). Expression of met-FTS by the ETV system in brain parenchyma needs to be tested. We believe that the difference in the duration of adenovirus-mediated transgene expression is likely to be due to normal ependymocyte turnover rather than to the immunogenicity of our ETV vector system. Therefore, the synthetic gene for metFTS appears as a potentially suitable therapeutic tool for the amelioration of pathologies involving 


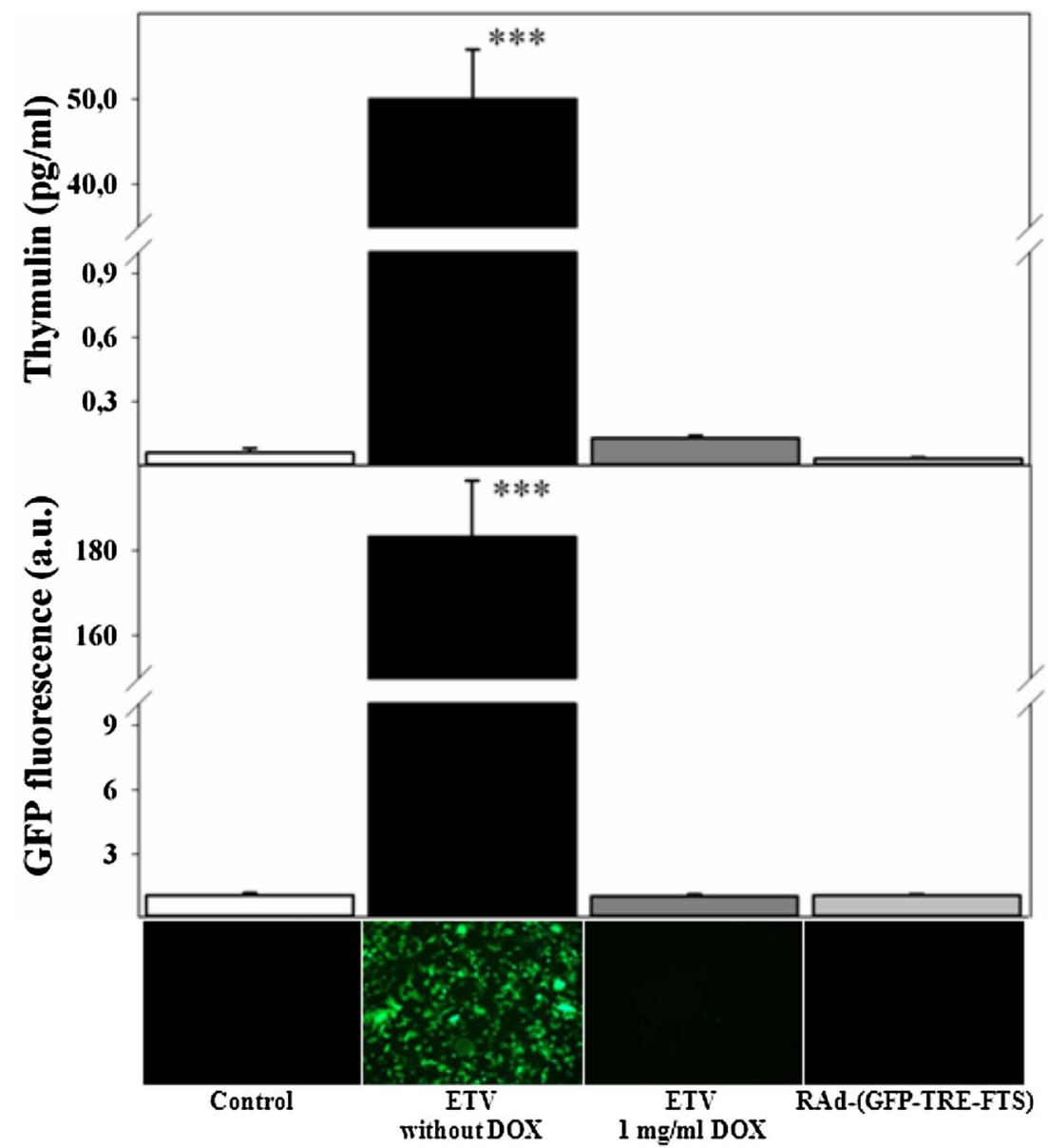

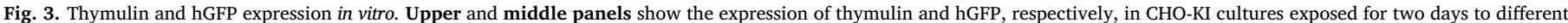

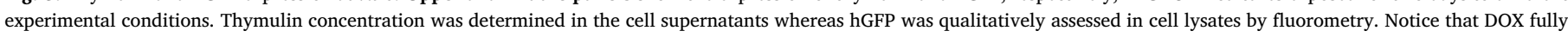

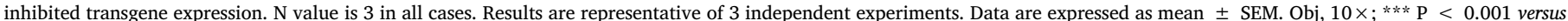
non-transduced control, analyzed by one-way ANOVA with post-hoc Tukey's test. The lower panel shows green fluorescence images of CHO-KI cells for each condition.

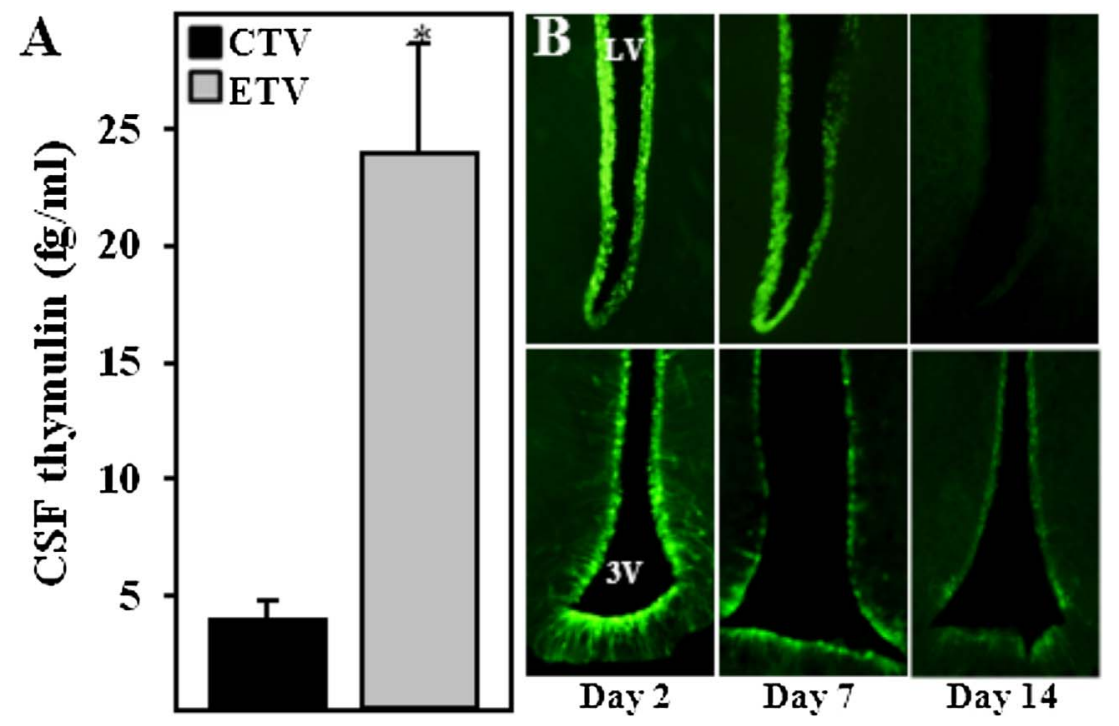

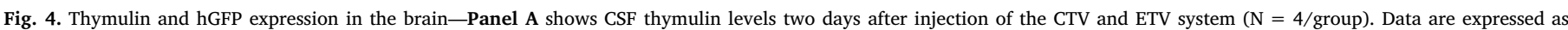

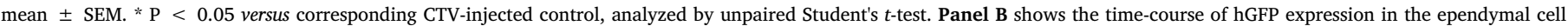

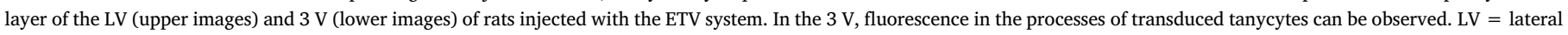
ventricle; $3 \mathrm{~V}=$ third ventricle. $\mathrm{Obj}, 20 \times$. 


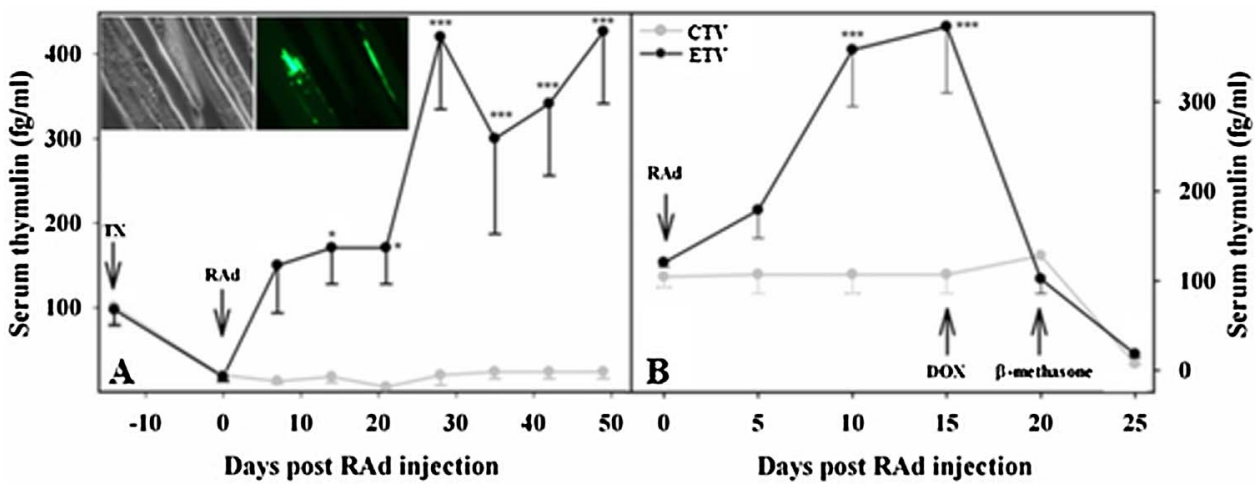

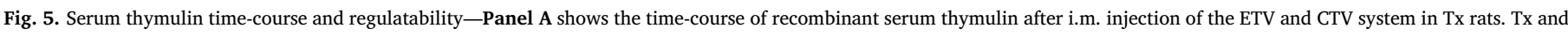

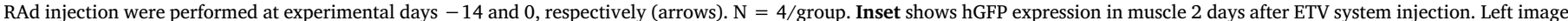

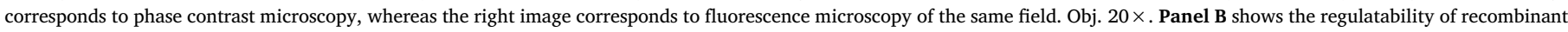

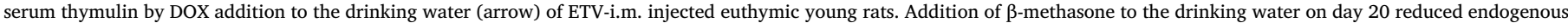

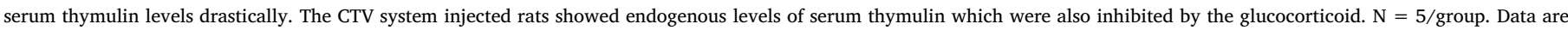
expressed as mean $\pm \mathrm{SEM} ; * \mathrm{P}<0.05 ; * * * \mathrm{P}<0.001$ versus corresponding CTV-injected time point, analyzed by two-way repeated measures ANOVA with post-hoc Sidak test.

acute and chronic brain inflammation.

The longer duration of thymulin expression in muscle is probably due to the anti-inflammatory activity of thymulin which prevented the immune system of the vector-injected animals from mounting a destructive response against the transduced muscle cells. The same rationale could explain the long-term persistence of high concentrations of recombinant metFTS in the circulation of RAd-metFTS-injected Tx rodents (Reggiani et al., 2006).

In this regard, it should be pointed out that the duration of transduction and transgene expression is shorter when the ETV system is delivered i.c.v., compared with the i.m. administration. We can only be certain that a markedly long-term expression of the met-FTS transgene occurs in muscle. This suggests that short-term i.c.v. thymulin gene therapy would be suitable for an acute inflammatory condition e.g. encephalitis. Whereas, based on its longer duration, i.m. administration would be the best choice for a long-term therapy, aimed at treating a rather chronic pathology. Expression of met-FTS in other tissues needs to be tested. In this context, the regulatable two-vector system that we have constructed constitutes an improved molecular tool for implementing thymulin gene therapy. In effect, the expression of the reporter hGFP makes it unnecessary to determine thymulin tissue levels for confirmation of adenovector functionality in the brain, muscle and any other tissue where the two-vector system is injected. This is an important advantage due to the fact that thymulin is a nonapeptide that easily binds to other proteins in tissues which generates considerable interference in thymulin assays in tissue extracts (Dardenne and Bach, 1975). Additionally, the reporter gene allows precise identification of the distribution of the transduced cells in the target tissue.

The regulatability of our two-vector system constitutes a second advantage for long-term treatments as it allows the gene therapist to manipulate therapeutic transgene expression during the treatment according of treatment needs. This manipulation is achieved by means of a noninvasive procedure (addition to or removal of DOX from the drinking water). Furthermore, in cell cultures, the regulatability of thymulin expression by DOX can be readily and noninvasively monitored by observing the changes in hGFP fluorescence in the treated cells. The tet system was first tested in a mammalian cell system (HeLa) by Gossen and Bujard (1992) who found that the system was functional, and had rapid induction with efficient tetracycline uptake. It proved to be quite appropriate for our transgenes.

We conclude that the two-vector system that we have constructed is likely to be suitable for short and long-term treatments in animal models (and eventually, humans) of brain and other pathologies where acute or chronic inflammation plays a relevant etiopathogenic role.

\section{Conflict of interest}

We hereby declare that there is no conflict of interest that could be perceived as prejudicing the impartiality of the research reported.

\section{Acknowledgements}

The authors are deeply indebted to the late Dr. Omar Rimoldi and to Dr. Jean-Marie Pleau for invaluable guidance for the molecular biology procedures. The authors are also grateful to Ms. Yolanda E. Sosa and Mr. Oscar Vercellini for technical assistance. RC, RGG, and PCR are career researchers of the Argentine Research Council (CONICET). MFZV, MLL, and JP are recipients of a CONICET doctoral fellowship.

This work was supported by grant \#PICT13-1590 from the Argentine Agency for the Promotion of Science and Technology (ANCYPT) to RGG as well as by grants \#PICT12-2461 and PIP0570 to PCR from ANCYPT and the Argentine Research Council (CONICET), respectively.

\section{References}

Bach, J.F., Dardenne, M., Goldstein, A.L., 1984. Clinical aspects of thymulin (FTS). In: Goldstein, A.L. (Ed.), Thymic Hormones and Lymphokines, Basic Chemistry and Clinical Applications. Plenum Press, New York, pp. 593-600.

Bach, J.F., 1983. Thymulin (FTS-Zn). Clin. Immunol. Allergy 3, 133-156.

Calenda, A., Cordonnier, A., Lederer, F., Le, D.K.H., Pleau, J.M., 1988. Production of biologically active thymulin in Escherichia coli through expression of a chemically synthesized gene. Biotechnol. Lett. 10, 155-160.

Cung, M.T., Marraud, M., Lefrancier, P., Dardenne, M., Bach, J.F., Laussac, J.P., 1988 NMR study of a lymphocyte differentiating factor. An investigation of the Zn(II)nonapeptide complexes (thymulin). J. Biol. Chem. 263, 5574-5580.

Da Silva, A.L., Martini, S.V., Abreu, S.C., Samary Cdos, S., Diaz, B.L., Fernezlian, S., de Sá, V.K., Capelozzi, V.L., Boylan, N.J., Goya, R.G., Suk, J.S., Rocco, P.R., Hanes, J., Morales, M.M., 2014. DNA nanoparticle-mediated thymulin gene therapy prevents airway remodeling in experimental allergic asthma. J. Control Rel. 180, 125-133.

Dardenne, M., Bach, J.F., 1975. The sheep cell rosette assay for the evaluation of thymic hormones. In: Van Bekkum, D.W., Kruisbeek, A.M. (Eds.), Biological Activity of Thymic Hormones, International Workshop and Symposium. Kooyker Scientific Publications, Halsted Press Division, Wiley, Rotterdam, pp. 235-243.

Dardenne, M., Papiernik, M., Bach, J.F., Stutman, O., 1974. Studies on thymus products. 3. Epithelial origin of the serum thymic factor. Immunology 27, 299-304.

Dardenne, M., Pléau, J.M., Nabarra, B., Lefrancier, P., Derrien, M., Choay, J., Bach, J.F., 1982. Contribution of zinc and other metals to the biological activity of serum thymic factor. Proc. Natl. Acad. Sci. U. S. A. 79, 5370-5373.

Gastinel, L.N., Dardenne, M., Pléau, J.M., Bach, J.F., 1984. Studies on the zinc-binding site to the serum thymic factor. Biochim. Biophys. Acta 797, 147-155.

Gossen, M., Bujard, H., 1992. Tight control of gene expression in mammalian cells by tetracycline-responsive promoters. PNAS 89, 5547-5551.

Goya, R.G., Cónsole, G.M., Hereñú, C.B., Brown, O.A., Rimoldi, O.J., 2002. Thymus and aging: potential of gene therapy for restoration of endocrine thymic function in thymus-deficient animal models. Gerontology 48, 325-328.

Goya, R.G., Cónsole, G.M., Spinelli, O.M., Carino, M.H., Riccillo, F., Corrons, F., 2003. Glucocorticoid-induced apoptosis in lymphoid organs is associated with a delayed 
increase in circulating deoxyribonucleic acid. Apoptosis 8, 171-177.

Goya, R.G., Brown, O.A., Pléau, J.M., Dardenne, M., 2004. Thymulin and the neuroendocrine system. Peptides 25, 139-142.

Goya, R.G., Reggiani, P.C., Vesenbeckh, S.M., Pléau, J.M., Sosa, Y.E., Cónsole, G.M., Schade, R., Henklein, P., Dardenne, M., 2007. Thymulin gene therapy prevents the reduction in circulating gonadotropins induced by thymulin deficiency in mice. Am. J. Physiol. Endocrinol. Metab. 293, E182-E187.

Henriques-Coelho, T., Oliveira, S.M., Moura, R.S., Boncon-Albuquerque, R.R., Neves Jr., A.L., Santo, M., Nogueira-Silva, C., La Fuente Carvalho, F., 2008. Thymulin inhibits monocrotaline-induced pulmonary hypertension modulating interleukin- 6 expression and suppressing p38 pathway. Endocrinology 149, 4367-4373.

Hitt, M., Bett, A., Prevec, L., Graham, F.L., 1998. Construction and propagation of human adenovirus vectors. In: Celis, J. (Ed.), Cell Biology: A Laboratory Handbook. Academic Press, New York, pp. 1500-1512.

Kimura, E., Maeda, Y., Arima, T., Nishida, Y., Yamashita, S., Hara, A., Uyama, E., Mita, S., Uchino, M., 2001. Efficient repetitive gene delivery to skeletal muscle using recombinant adenovirus vector containing the Coxsackievirus and adenovirus receptor cDNA. Gene Ther. 8 (1), 20-27.

McGeer, P.L., McGeer, E.G., 2004. Inflammation and neurodegeneration in Parkinson's disease. Parkinsonism Relat. Disord. 10, S3-S7.

Morel, G.R., Brown, O.A., Reggiani, P.C., Hereñú, C.B., Portiansky, E.L., Zuccolilli, G.O., Pléau, J.M., Dardenne, M., Goya, R.G., 2006. Peripheral and mesencephalic transfer of a synthetic gene for the thymic peptide thymulin. Brain Res. Bull. 69, 647-651.

Nishida, F., Morel, G.R., Hereñú, C.B., Schwerdt, J.I., Goya, R.G., Portiansky, E.L., 2011. Restorative effect of intracerebroventricular Insulin-like Growth Factor-I gene therapy on motor performance in aging rats. Neuroscience 177, 195-206.

Pardo, J., Uriarte, M., Console, G.M., Reggiani, P.C., Outeiro, T.F., Morel, G.R., Goya,
R.G., 2016. Insulin-like growth factor-I gene therapy increases hippocampal neurogenesis, astrocyte branching and improves spatial memory in aging rats. Eur. J. Neurosci. 44, 2120-2128.

Paxinos, G., Watson, C., 1998. The Rat Brain in Stereotaxic Coordinates. Academic Press, San Diego.

Reggiani, P.C., Hereñú, C.B., Rimoldi, O.J., Brown, O.A., Pléau, J.M., Dardenne, M., Goya, R.G., 2006. Gene therapy for long-term restoration of circulating thymulin in thymectomized mice and rats. Gene Ther. 13, 1214-1221.

Reggiani, P.C., Barbeito, C.G., Zuccolilli, G.O., Cónsole, G.M., Flamini, A.M., Dardenne, M., Goya, R.G., 2012. Neonatal thymulin gene therapy prevents ovarian dysgenesis and attenuates reproductive derangements in nude female mice. Endocrinology 15, 33922-33928.

Safieh-Garabedian, B., Kendall, M.D., Khamashta, M.A., Hughes, G.R.V., 1992. Thymulin and its role in immunomodulation. J. Autoimmun. 5, 547-555.

Safieh-Garabedian, B., Ochoa-Chaar, C.I., Poole, S., Massaad, A., Atweh, S.F., Jabbur, S.J., 2003. Thymulin reverses hyperalgesia and modulates the increased concentration of pro-inflammatory cytokines by i.c.v. endotoxin injection. Neuroscience 121 , 865-873.

Safieh-Garabedian, B., Jabbur, S.J., Dardenne, M., Saadé, N.E., 2011. Thymulin related peptide attenuates inflammation in the brain induced by intracerebroventricular endotoxin injection. Neuropharmacology 60, 496-504.

Waynforth, H.B., Flecknell, P.A., 1992. Specific surgical operations. Thymectomy. Experimental and Surgical Technique in the Rat, second ed. Academic Press, London, pp. 304-308.

Yamanouchi, T., Moromizato, H., Kojima, S., Shinohara, T., Sekino, N., Minoda, S., Miyashita, H., Akaoka, I., 1994. Prevention of diabetes by thymic hormone in alloxan-treated rats. Eur. J. Pharmacol. 25, 739-746. 